\title{
Desarrollo de la creatividad en la primaria a partir del cuento musical
}

\author{
Creativity development in primary school throughout musical story
}

Desenvolvimento da criatividade na primária do conto musical

DOI: http://dx.doi.org/10.23913/ride.v7i14.284

\author{
Nadezhda Borislavovna Borislova \\ Benemérita Universidad Autónoma de Puebla, México \\ borislavovna.borislova@correo.buap.mx
}

\section{Resumen}

En años recientes se puede observar un creciente interés por estudiar a las personas creativas desde distintos enfoques y perspectivas, así como el rol y la función que la escuela tiene en todos los niveles educativos para el desarrollo de la creatividad. La creatividad es abordada y estudiada no solamente por los psicólogos, también por educadores, artistas, filósofos, biólogos, empresarios, entre otros. En las primeras dos décadas del siglo XXI, la creatividad se ha vuelto un sinónimo de educación, porque existe una necesidad urgente de educar a nuestros niños y jóvenes de una forma creativa, cambiar los esquemas convencionales por los esquemas del pensamiento divergente, creativo y original que incentive el estudio y desarrollo de la creatividad.

Escuchar el cuento musical como parte de las actividades de la educación artística dentro del currículo escolar, es una herramienta muy valiosa para el desarrollo de este potencial. El análisis de los resultados del estudio realizado con 582 niños de $1^{\circ}$ a $6^{\circ}$ grado de una escuela primaria pública de la ciudad de Puebla, conduce a la necesidad de incluir el cuento musical como parte del currículo de la educación artística. A pesar de una gran variedad del acervo literario-musical que nos ha dejado la historia, sobre todo en los últimos años. El cuento musical, por diversas razones, aún no ha sido explorado y utilizado con fines pedagógicos en 
la educación primaria. Por lo tanto, este trabajo plantea la necesidad de generar líneas de investigación específicas, enfocadas al estudio y desarrollo de la creatividad a partir de las artes que permitan el desarrollo integral de los niños en la educación básica a nivel primaria, al demostrarse que el cuento musical grabado motivó una transformación estadísticamente considerable del promedio de puntaje de creatividad (mismo que se incrementó de forma estadísticamente significativa en el post-test) en los grados $1^{\circ}, 2^{\circ}, 4^{\circ}$ y $5^{\circ}$ en específico, y al juntar a todos los niños de todos los grados $\left(1^{\circ}\right.$ al $\left.6^{\circ}\right)$ que tomaron el test después del cuento musical.

Palabras clave: cuento musical, creatividad en la primaria, cuento y música.

\section{Abstract}

In recent years we can see a growing interest in studying creative people from different viewpoints and perspectives, as well as the role and function of the school at all educational levels for the development of creativity. Creativity is approached and studied not only by psychologists, but also by educators, artists, philosophers, biologists, entrepreneurs, among others. In the first two decades of the 21 st century, creativity has become synonymous of education, because there is an urgent need to educate our children and young people in a creative way, to change conventional schemes by divergent, creative and original thinking schemes which encourages the study and development of creativity.

The development of creativity depends on the opportunities the child has to experiment with his fantasy and imagination. Listening to the musical story as part of artistic education activities within the school curriculum is a very valuable tool for the development of this potential. The results analysis of the study carried out with 582 children from 1 st to 6 th grade of a public primary school in the Puebla city, leads to the need to include the musical story as part of the curriculum of artistic education. Despite a great variety of literarymusical heritage that has left us the history, especially in recent years, the musical story, for various reasons, has not yet been explored and used for educational purposes in elementary education. Therefore, this work raises the need to generate specific research lines, focused on the study and development of creativity based on the arts that allow the integral children 
development in basic education at primary level, demonstrating that the recorded musical story motivated a statistically significant transformation of the average of the creativity score (which increased in a statistically significant way in the post-test) in grades 1,2, 4 and 5 in specific, and bringing together all The children of all grades (1st-6th) who took the test after the musical story.

Key words: musical stories, creativity at primary school, stories and music.

\section{Resumo}

Nos últimos anos, pode-se observar um crescente interesse em estudar pessoas criativas de diferentes abordagens e perspectivas, bem como o papel e função que a escola tem em todos os níveis educacionais para o desenvolvimento da criatividade. A criatividade não só é abordado e estudado por psicólogos, também por educadores, artistas, filósofos, biólogos, empresários, entre outros. Nas duas primeiras décadas do século XXI, a criatividade se tornou sinônimo de educação, porque há uma necessidade urgente de educar nossos filhos de uma forma criativa, alterar os esquemas convencionais por esquemas de pensamento divergente, criativa e original que incentiva o estudo e desenvolvimento de criatividade.

Ouça o conto musical como parte das atividades de educação artística no currículo escolar, é uma ferramenta muito valiosa para o desenvolvimento deste potencial. A análise dos resultados do estudo com 582 crianças de $1^{\mathrm{a}}$ a $6^{\mathrm{a}}$ série de uma escola pública na cidade de Puebla, leva à necessidade de incluir o conto musical como parte do currículo da educação artística. Apesar de uma variedade de herança literária e musical que nos deixou a história, especialmente nos últimos anos. O conto musical, por diversas razões, ainda não foi explorado e utilizado para fins educacionais no ensino primário. Portanto, este artigo discute a necessidade de desenvolver linhas específicas de investigação incidiu sobre o estudo e desenvolvimento da criatividade das artes que permitam o desenvolvimento integral das crianças na educação básica no nível elementar, para provar que a história música gravada levou a uma criatividade médio estatisticamente significativa ponto de transformação (o mesmo que aumentou de forma estatisticamente significativa no pós-teste) em graus 1, 2, 4 e 
5, em particular, e para reunir todos os filhos de todos notas ( 1 a 6 ) que fizeram o teste depois que a história musical.

Palavras-chave: conto musical, criatividade no ensino primário, história e música.

Fecha Recepción: Mayo $2016 \quad$ Fecha Aceptación: Octubre 2016

\section{Introducción}

En los últimos años, la creatividad ha sido abordada y estudiada no solamente por psicólogos, también por educadores, artistas, filósofos, biólogos, empresarios, entre otros. Al respecto, Gardner (2011) postula la necesidad de la investigación interdisciplinaria. Este interés crece cada día y nos confirma la importancia y la actualidad del tema, que se ha vuelto primordial en todos los campos del quehacer humano. Esta visión se ha tornado como necesidad del siglo XXI, siendo un sinónimo de educación. Waisburg y Erdmenger (2008) resaltan como responsabilidad social el motivar y estimular la creatividad dentro del currículo escolar, siendo más significativo en el desarrollo del niño comprendido entre los seis a doce años, al ser, como dice Vigotsky (1984), un periodo determinante para la vida futura.

Vigotsky (2008) al igual que muchos otros autores (Maslow, 1982; Csikszentmihalyi, 1998; Hargreaves, 1998; Campbell, 2007, 2011; Copland, 2003) enfatiza que parte de la creatividad depende de las oportunidades que el niño tiene para experimentar con su fantasía e imaginación, no "como entretenimiento festivo de la mente o una actividad detenida en el aire, sino como una función vital e indispensable” (p. 505).

Sustentando lo anterior, Bertomeu (2006) recalca el hecho de que "los niños y jóvenes en contacto con el arte, la literatura y la música liberan su imaginación, su sensibilidad y su creatividad” (p. 54). En tanto, autores como López Romero (2006a) especifican qué cuentos estimulan la imaginación infantil, porque "no todos los cuentos estimulan la creatividad y la sensibilidad artística" (p. 146). 
Si la creatividad depende de la fantasía e imaginación del niño y éstas nacen gracias a su experiencia, la implementación del cuento musical como parte de las actividades de la educación artística u otras materias, puede ser una valiosa herramienta para el desarrollo de dicho potencial, especialmente dentro del currículo escolar.

El cuento musical cuenta con una relación íntima no solamente con la música - la cual forma parte de la educación artística-, sino con otras materias, por ejemplo el Español. Esta actividad puede ser un soporte significativo para el desarrollo de la creatividad, así como para el del pensamiento y las habilidades lingüísticas.

Orff-Schulwerk fue uno de los métodos pioneros en destacar la importancia de usar la palabra (hablada o escrita) en la educación musical, ya que representa "un mundo inigualable de expresión" (Orff-Schulwerk, 1969, pp. 12-13), puesto que antes de cualquier ejercicio musical, ya sea melódico o rítmico, existe el ejercicio de hablar.

A pesar de que muchos investigadores han realizado y publicado estudios en el ámbito del cuento y de la música, hay temas de gran interés que no han sido tratados todavía. El cuento musical, por diversas razones, aún no ha sido explorado y utilizado — con fines pedagógicos y de manera generalizada - en la educación primaria. Es por ello que investigar el efecto que el cuento musical pudiera tener en la capacidad creativa de alumnos a nivel escolar de primaria resulta sumamente importante.

\section{LA CREATIVIDAD Y SUS ELEMENTOS CONSTITUTIVOS}

Según López Pérez (1999) y otros investigadores como Tatarkiewicz (1987), en el mundo antiguo el concepto de la creatividad, tal como lo conocemos hoy, no existió sino hasta el siglo XVII, cuando aparece "tímidamente" debido a la sensibilidad religiosa, privando al hombre de la concepción de crear.

La mayoría de las referencias al término Creatividad corresponden a nuestra época. Tal vez, como dice Romo (1997), el interés por el estudio de la creatividad y capacidad innovadora se debe, en buena parte, a razones de índole política: "el llamado Shock del Esputnik 
[Sputnik] $]^{1}$, un sentimiento de fracaso nacional ante la primera salida al espacio que fue soviética y no norteamericana, influyó también sobre la toma de conciencia de la necesidad de mayor capacidad innovadora entre los cientificos de la NASA" (p. 7). Es posible que el gobierno estadounidense se haya preocupado por este hecho, haciendo una serie de interrogaciones sobre la importancia de la capacidad creativa como parte de las capacidades intelectuales.

Hargreaves (1998), Wilson (1997), Waisburg y Erdmenger (2008) refieren a la creatividad como un elemento de complejidad y misterio, polisémico en el sentido de que su propio significado es creativo. Guilford en la década de los cincuenta, expresa la necesidad de estudiar la creatividad como una disciplina más de la psicología, revelando la complejidad del fenómeno, siendo necesario para su comprensión diferentes niveles o "claves de la creatividad", según López de la Llave y Pérez (2006):

- Sensibilidad. La persona creativa es sensible y capaz de identificar los problemas, necesidades, actividades y sentimientos de los otros; además, percibe todo lo inusual o prometedor que poseen las personas, los objetos o las situaciones con las que se relaciona.

- Fluidez. Consiste en la producción de un gran número de respuestas en una situación, es la fertilidad de ideas y de conductas.

- Flexibilidad. Es la capacidad del individuo para organizar los hechos dentro de diversas y amplias categorías. Es la capacidad de modificación, de variación en comportamientos, actitudes, objetos, objetivos y métodos.

- Originalidad. Se manifiesta a través de las respuestas "no comunes" que pueden darse en determinada situación. Lo original se reconoce por apartarse de lo habitual, por su singularidad.

\footnotetext{
${ }^{1}$ Sputnik se refiere al primer satélite artificial de la historia, el cual fue lanzado el 4 de octubre de 1957 por la Unión Soviética.
} 
- Redefinición. Es la capacidad para reacomodar ideas, conceptos, personas y cosas, para transponer las funciones de los objetos y estímulos de maneras novedosas; se refiere a la habilidad para definir o percibir los objetos o las situaciones de manera distinta a la usual.

- Análisis. Permite extraer detalles de la totalidad.

- Síntesis. Es la habilidad de combinar varios componentes para llegar a una totalidad.

- Divergencia. Es la capacidad del individuo para analizar lo opuesto, para visualizar lo diferente, para encontrar caminos diferentes.

- Elaboración. Es la capacidad del individuo para formalizar las ideas, para planear, desarrollar y ejecutar proyectos. Es la actitud para convertir las formulaciones en acciones decisivas, es la exigencia de llevar el impulso creativo hasta su realización.

- Utilidad. Una idea, un objeto o un proceso creativo debe ser capaz de resolver algún problema, de proponer un nuevo enfoque a alguna cuestión pendiente. Sólo se reconoce algo como creativo si resulta ventajoso individual o socialmente útil (en el más amplio sentido de este concepto) (p. 61).

Siguiendo a este autor, es "imposible alcanzar un concepto como el que se pretende, esto es, simple en su expresión, pero lleno de contenido" (p. 17). Sin embargo, destacan algunas definiciones de uso habitual como “asociaciones nuevas que son útiles” (Firestein, 1991), por ejemplo, explica la creatividad como la "capacidad para producir cosas nuevas y valiosas" (Rodríguez, 2009), la "capacidad de revelar nuevas relaciones, cambiar las normas existentes de manera razonable y contribuir así a la resolución general de problemas en la realidad social” (Wollschlager, 1976). "Creatividad es apartarse del camino principal, romper el molde, estar abierto a la experiencia y permitir que una cosa lleve a la otra" (Bartlett, 1995). "La creatividad consiste en formar combinaciones nuevas de elementos viejos. Estas combinaciones tienen que observar determinados requisitos o ser útiles de alguna manera. Cuanto más disten entre sí los elementos, más creativa resultará la 
combinación" (Mednick, 1964). Por añadidura, Torrance (1970) habla de ella como proceso de apreciación de problemas, formación de ideas, verificación de hipótesis con la correspondiente comunicación de resultados, destacando los elementos de conectividad, originalidad y relevancia que enuncia López. Frega (2009) así como Waisburg y Erdmenger (2008) y Csikszentmihalyi (1998) hacen referencias a entornos creativos. A partir de aquí, podemos hablar de criterios de la creatividad.

\section{Criterios de la creatividad}

1. La energía creativa es la actividad intelectual que realiza el producto (en esto coincide con Mihaly Csikszentmihalyi en su libro Creatividad. El fluir y la psicología del descubrimiento y la invención (1998). Además, esta energía coincide con la energía sexual y el reposo creador en sus diez contrastes o paradojas con que define la creatividad a partir de una metodología basada en entrevistas a grandes creadores.

2. La creación es útil, tiene utilidad (es más complejo definir la utilidad en el arte).

3. Tiene novedad. Superando las dificultades, la respuesta de la inventiva es una sorpresa; el mismo creador se puede sorprender.

4. La experimentación precede a la realización de la obra o producto.

5. La inventiva se presenta en el producto exitosamente terminado, en un área poco común.

6. Hay escepticismo previo de los colegas en esa área activa ante un nuevo producto científico (aunque hay rasgos generales comunes entre la creatividad científica y la artística, creemos que un estudio comparativo profundo entre las dos áreas de la creación humana sería muy útil, incluso entre las distintas ramas y especialidades de cada creación).

7. En el individuo hay una insatisfacción ("un deseo incumplido") realizada después con el nacimiento de la novedad productiva (p. 75). 
Partiendo de múltiples enfoques sobre la creatividad, se afirma que la creatividad abarca cuatro conceptos: persona, proceso, producto y ambiente creativo. La Creatividad es la capacidad humana (persona) de producir obras —u obra (producto) — originales o nuevas, las cuales son el resultado de un proceso creativo de fluir de la creatividad (término introducido por Csikszentmihalyi, 1997). Se crea en un entorno (ambiente y público), que puede emitir distintos juicios (favorables o de rechazo) respecto al producto creativo. Por lo expuesto, es necesario revisar cada uno de estos enfoques por separado.

\section{Persona creativa}

Según Csikszentmihalyi (1998), las personas creativas se adaptan a cualquier situación y alcanzan sus objetivos con lo que tengan a la mano (p. 73). Aun cuando es difícil definir rasgos específicos o características de individuos creativos, éstos pueden ser muy sociales y optimistas, pero también pueden ser antisociales y depresivos, o muy generosos y alegres, como también avaros y amargados, soberbios o tímidos. Al mismo tiempo, estas características pueden ser engañosas, porque son las opiniones o impresiones de otros.

La personalidad creativa es un asunto sumamente complejo, ocupando un lugar central en los trabajos de Csikszentmihalyi, quien le dedicó varios de sus escritos, entre ellos The Evolving Self: A Psychology for the Third Millennium. En 1996 (1998 en español), publica Creativity. Flow and the Psychology of Discovery and Invention, en donde el autor entrevista a noventa y una personalidades creativas de todo el mundo, las cuales han revolucionado sus áreas de trabajo. En uno de sus capítulos dedicado a la personalidad creativa, Csikszentmihalyi describe “las diez dimensiones de la complejidad” (pp. 78-99), directrices que se resumen a continuación:

\section{Los diez pares contrastantes de la personalidad creadora ${ }^{2}$}

1. Tienen mucha energía y capacidad de trabajo, igual que "parecen tener una dosis muy alta de eros o energía libidinal generalizada, que algunos expresan directamente en la sexualidad". Pero también se encuentran callados y en reposo, trabajando muchas horas concentrados, con frescura y

\footnotetext{
${ }^{2}$ Se decidió por este título, porque cada una de las diez dimensiones de la complejidad de las personas creativas que describe Csikszentmihalyi implican siempre dos fenómenos o características contrastantes de la personalidad creativa.
} 
entusiasmo, dotación física superior y ventaja genética. Sin embargo es frecuente que septuagenarios creativos puedan haber tenido una infancia llena de enfermedades. Actividad febril y descanso flexibles son claves en la creación (pp. 80-82).

2. Los individuos creativos son "vivos" e ingeniosos a la par que ingenuos (añadiríamos paradójicamente "maliciosos", Goethe decía que "la ingenuidad es el atributo más importante del genio”, recordemos que genio viene de «gen». De manera que genitivo, generar, ingenuo y genio tienen una raíz común. Se sabe que los índices de Coeficiente Intelectual (CI ) no son confiables, tampoco directamente concluyentes, respecto del individuo creativo, sin embargo, la creatividad requiere un mínimo de 120 de coeficiente intelectual. Pese a todo, no hay un test que mida claramente los distintos tipos de creatividad, pues una persona creativa une el pensamiento divergente con el convergente, es decir, una flexibilidad con rigor a su vez que conjunta la intuición con el buen juicio. (pp. 82-84).

3. Lo inmediatamente anterior nos lleva al tercer rasgo creativo, el cual combina el carácter lúdico con la disciplina. El ser creativo posee humor y poder del juego: es irresponsable y responsable al mismo tiempo, pero como decíamos: su constancia en el perfeccionamiento de una idea creativa es fundamental. (pp. 84-86).

4. La imaginación y la fantasía por un lado y un profundo sentido de la realidad por otro, es característica de la personalidad creativa. El arte y la ciencia son formas sublimes de escapar de la realidad, decía Einstein. Lo esencial de los individuos creativos es ir más allá de la realidad creando una nueva, también verdadera. La novedad de ellas está enraizada en la realidad, no son extravagantes, no divagan, son coherentes: están estructurados. En cambio constante, la realidad es relativa - dice una famosa mente creativa financiera, haciendo un eco einsteniano-; la realidad no está vacía pero anda cerca de estarlo. Desde su punto de vista, el éxito es evolutivo. (pp. 86-88). 
5. Las personas creativas son introvertidas y extrovertidas (continúan sus paradojas). "Sólo aquellos que pueden estar solos son capaces de dominar el contenido simbólico de un campo”, pero también insisten en la importancia del diálogo con la gente. Solitarios y sociables, establecen relaciones creando, y crean solitariamente. (pp. 88-90).

6. La personalidad creativa es humilde y orgullosa, autocrítica y de gran confianza en sí misma; dependiendo del medio, es agresiva si es necesario. Son autocríticos porque se centran sobre todo en su actividad presente. Una persona famosa no es necesariamente arrogante, puede ser tímida por ser autocrítica, consciente de las contribuciones de quienes les precedieron - se sitúan en perspectiva—, del papel que les asignó también la suerte, centrados en el presente y el futuro, sus esfuerzos pasados no les son particularmente interesantes. Pueden estar más orgullosos de su papel como padres que de su reputación. Frecuentemente, subordinan bienestar y promoción al éxito del proyecto que los entusiasma. Pacíficos y agresivos, según las circunstancias, varios pasan de ser egocéntricos a ser altruistas, sobre todo en la madurez. (pp. 90-93).

7. Los hombres y mujeres creativos trascienden los estereotipos de su género. Esta androginia psicológica no se debe confundir con la homosexualidad. Son agresivos y protectores, sensibles y rígidos, dominantes y "sumisos". La creatividad une las fuerzas y virtudes de los dos cerebros (el "masculino" y el "femenino"). Los hombres creativos tienen gran preocupación por su familia y sensibilidad por aspectos sutiles del entorno, como rasgos "femeninos", las mujeres talentosas son más dominantes y duras que el común de ellas, pero en general los hombres son perfectamente masculinos como las mujeres absolutamente femeninas, poseyendo rasgos del género opuesto. (pp. 93-94)

8. No se puede jugar un juego sin conocer sus reglas: no se puede ser creativo (y rebelde, e iconoclasta) sin ser tradicional y conservador. Para ser creativo, hay que interiorizar primero un campo de la cultura. Según la artista Eva Zeisel, ninguna creatividad surge de un impulso negativo (lo que le pasa al 
posmodernismo, según ella); en otras palabras, solamente el impulso positivo produce una creación satisfactoria. Al mismo tiempo, el creador rompe con la seguridad de la tradición, pero sobre la base de su profundo conocimiento. (pp. 94-95)

9. La gran pasión por su trabajo es una característica de los seres creativos como — paralelamente - la objetividad por éste. Sin pasión se pierde pronto el interés. Sin objetividad, el trabajo carece de credibilidad pues no será muy bueno. El proceso creativo es una alternancia entre los dos extremos.

10. El último de los pares contrastantes de la personalidad creativa, según el autor, es la apertura y sensibilidad del creador que lo expone al sufrimiento como a un gran placer. Estar en la vanguardia lo deja desprotegido y vulnerable, la eminencia invita a la crítica y a los ataques virulentos. El pensamiento divergente constantemente es entendido por la mayoría como una desviación, lo que puede hacer que el creador se sienta incomprendido, pero lo más difícil es la sensación de vacío y de pérdida cuando no puede trabajar. Pese a ello, cuando está trabajando en su especialidad hay una sensación de dicha. Una constante en este tipo de personalidad es la de disfrutar el proceso creativo en sí mismo. La atención dividida, la entrega a medias, no es compatible con la creatividad, los creadores disfrutan generalmente también con muchas otras actividades que realizan. (pp. 96-99)

Además, Csikszentmihalyi garantiza: “Una persona no puede ser creativa en un campo en el que no ha sido iniciada. Por enormes que sean las dotes matemáticas que pueda tener un niño, no será capaz de hacer una aportación a las matemáticas sin aprender sus reglas” (p. 47). Por otra parte, el desarrollo de todas estas habilidades estarán sujetas al "ámbito que reconozca y legitime las aportaciones novedosas" (Csikszentmihalyi, 1998, p. 47). El tema del ámbito se abordará más adelante. 


\section{El proceso creativo}

El proceso creativo exige un trabajo constante. Vigotsky lo confirma: "crear es dificil". Incluso, trata este tema en el capítulo Los sufrimientos de la creación, del libro Pensamiento y lenguaje (Vigotsky, 2008, pp. 535-538). Además, Csikszentmihalyi (1998) se refiriere al proceso creativo utilizando las palabras de Thomas Edison, quien decía: "la creatividad consiste en $1 \%$ de inspiración y un 99\% de transpiración" (p. 104). Hargreaves (1998) describe el proceso creativo con las palabras del cantante Sammy Kahn: "Cuando la gente me pregunta qué viene primero, si la música o la letra, les digo que lo que viene primero es la llamada telefónica" (como se cita en Hargreaves, 1998, p. 163). El trabajo artístico demanda un trabajo duro y persistente.

Con este ejemplo del cantante estadounidense, se constata que el proceso creativo culminará en una obra y lo que finalmente le interesa al artista es el producto final, es decir, para el caso de cantautor, la canción que va a entregar. Con los niños no sucede lo mismo. Para ellos no es tan importante el producto final, sino el proceso de producción (Gardner, 1994, p. 47). También Vigotsky (2008) refiere algo al respecto: "No hay que olvidar que la regla principal de la creatividad infantil consiste en que su valor se encuentra no en el resultado o en el producto de su creación, sino en el proceso mismo" (p. 571). En esa misma línea de ideas, Viktor Lowenfeld destaca la importancia de hacer arte, por encima del producto final interesándole fundamentalmente lograr el desarrollo de la capacidad creadora y mental de los alumnos por medio de las actividades artísticas. Postuló que eran seis las esferas de crecimiento que sufrían la influencia de dichas actividades: emocional, física, perceptual, social, estética y creadora (como se cita en Wilson, 1979, pp. 13-14).

Además, señala que las técnicas artísticas no pueden enseñarse ni explicarse, sino que cada niño debe crear sus propios recursos para ejecutar su tarea, siendo que la expresione de creatividad infantil que atrae más al niño es la actuación teatral (Vigotsky, 2008, p. 568). Vigotsky describe el juego como la forma primaria de dramatización, por lo que es de vital importancia para la formación del carácter y la visión del mundo en el niño (p. 570). El autor de Pensamiento y lenguaje comenta que el niño puede ser mal actor para los demás y perfecto para él mismo (Vigotsky, 2008, p. 572). Esta característica afirma una vez más que al niño no le importa el resultado, sino el proceso. La mejor recompensa por la realización 
de la obra teatral debe ser el placer que siente el niño de participar en todos los procesos relacionados con la obra teatral, desde el proceso preparativo (pintar, esculpir, cocer, hacer decoraciones, aprender sus líneas, ensayar y demás) hasta el momento de actuar (Vigotsky, 2008, p. 570).

De acuerdo con Csikszentmihalyi (1998), el proceso creativo consta de cinco pasos o fases:

1. Período de preparación o inmersión.

2. Período de incubación.

3. Intuición: el momento jajá!

4. Período de autocrítica: la evaluación.

5. Proceso de la elaboración.

Este proceso o "estructura clásica" - como le denomina el autor - comienza con el período de preparación y culmina con el proceso de elaboración. No obstante, no hay que tomarlo demasiado literal:

Una persona que hace una aportación creativa nunca se limita a trabajar afanosamente durante el largo estadio último de elaboración. Esta parte del proceso está interrumpida constantemente por períodos de incubación y salpicada por pequeñas epifanías. Son muchas las intuiciones nuevas que surgen mientras, presuntamente, sólo se están dando los toques finales a la intuición inicial [...] Así, el proceso creativo no es tanto lineal como recurrente. El número de iteraciones por las que pasa, de vueltas que encierra, de intuiciones que precisa, es algo que depende de la profundidad y amplitud de los temas que se tratan. A veces la incubación dura años; a veces, unas horas. A veces la idea creativa incluye una intuición profunda y un número incalculable de otras menores. (Csikszentmihalyi, 1998, p. 105) 
Según los estudios de Guilford (1980) y Torrance (1969), entre otros especialistas, en el proceso creativo participan dos tipos de pensamiento: divergente y convergente. López (1999) dice que el pensamiento divergente es equivalente "a mirar desde distintas perspectivas. Es por sobre todo un pensamiento que no se restringe a un plano único...", mientras que el pensamiento convergente "se emplea para resolver problemas bien definidos cuya característica es tener una solución única.” (p. 75).

\section{Producto creativo}

Si bien persona y proceso creativo aluden a algo intangible, el producto creativo se refiere a cosas concretas que logramos ver, tocar o escuchar. Waisburg y Erdmenger (2008) lo definen como "[...] la constatación del proceso creativo que se comparte con los otros. Es lo que hace visible lo invisible, la creatividad es un proceso interno que se comprueba al hacerlo externo" (p. 62). A su vez, López (1999) apunta: "En la categoría de producto se consideran los criterios que hacen que una obra, objeto o idea puedan ser calificados de creativos, y los antecedentes que permiten establecer niveles de la creatividad o formas de manifestación de la conducta creadora" (p. 10).

La creación implica un producto, el verbo crear es transitivo. Según Romo (1997), lo espectacular no es el proceso sino el resultado, pero entendemos que ésta es una especie de abstracción, debido a que el proceso de la creatividad puede ser también espectacular. La autora llama a este proceso insight (que puede entenderse como inspiración, intuición, conexión, chispazo, apertura o inicio, impulso del momento creativo, entre otras). Para la autora, la sincronicidad está muy relacionada con la creatividad, y logra provocar o conducir a la creatividad. La invención puede tener estructuras sincrónicas-sincronizadas, por ejemplo, en la música o la poesía una imagen está tejida con el ritmo: es la imagen del ritmo y el ritmo de la imagen, y a su vez estas dos instancias implican una cosmovisión (p. 65). Desde el físico Linus Pauli, hasta Jung, Ortiz Osés, Neumann, Eliade, Durand, Kawai y otros teóricos del Círculo de Eranos, han estudiado la creatividad —principalmente artística - en relación profunda con ella, la hermenéutica y la simbología donde, insistimos, el proceso y no sólo el resultado es espectacular.

Donald MacKinnon (1975) indica que los productos creativos son la base sobre la cual descansa toda creatividad pero dicho fundamento debe estar firmemente construido. 
Debemos tener claro lo que constituye una obra creativa para construir una psicología de la creatividad. Ronald Finke (1992) dice que hay algo especial en el gran arte. Más allá de un consenso, contiene algo esencial acerca de la naturaleza del hombre. "Lo social puede incidir en el producto pero es irrelevante en la determinación del proceso porque, en todo caso, va a intervenir largo tiempo después que el producto haya surgido de la mente del creador" (como se cita en Romo, 1997, pp. 67-68).

Como hay objetos de los que se puede decir definitivamente que son productos creativos, se puede aplicar el método científico al estudio de la creatividad. El trabajo pionero de Gruber sobre Darwin (1974) marcó un hito en la explicación de la creatividad desde la psicología cognitiva. El método surgido se llamó Estudio Cognitivo de Casos. Gardner (2011), cognitivista también, estudió a Gandhi y a Picasso, entre varias muestras distintas. "El valor inductivo de los datos de observación obtenido en un riguroso estudio de casos tiene suficiente identidad como para plantear modelos explicativos de la creatividad, como el tan celebrado 'sistemas en evolución' de Gruber" (Romo, 1997, p. 69).

Por lo tanto, Romo busca definir qué productos son creativos y explicar por qué las cosas de la creatividad contienen novedad y valor. El producto cobra una existencia independiente del creador para trasmitirse, es comunicable. En la ciencia, la idea debe comprobarse, "en una contrastación empírica con los valores propios del sistema conceptual donde surgió" (Romo, 1997, p. 73).

Newell, Shaw y Simon (1958) sugieren los siguientes criterios que se deben satisfacer mínimamente para considerar creativo el producto:

1. El producto tiene novedad para el pensador y la cultura.

2. No es convencional, modificó o rechazó ideas aceptadas previamente.

3. El producto resulta de una gran motivación y persistencia, con intenso trabajo y gasto de tiempo (la Energía Creativa de Csikszentmihaly).

4. El producto puede ser el resultado de la formulación de un problema más formulado o definido. "No hay problemas en realidad, lo que hay son problemas mal planteados, cuando se plantea correctamente un problema 
éste se resuelve automáticamente”, diría Ludwig Wittgenstein (como se cita en Romo, 1997, pp. 75-76).

\section{Ambiente creativo}

Como arguye Csikszentmihalyi (1998) "la creatividad está construida conjuntamente por la interacción entre campo, ámbito y persona" (p. 47).

A partir de las tres categorías de Csikszentmihalyi (1998): la persona o el talento individual; el campo o la disciplina; y el ámbito; Gardner forma un armamentario para su estudio, como "un trío de amplios temas" que lo guiaron a lo largo de su investigación sobre los siete grandes creadores: Freud, Einstein, Picasso, Stravinsky, Eliot, Graham y Gandhi (Gardner, 2011, p.74). Los resultados de este estudio están publicados en el libro Mentes creativas. Una anatomía de la creatividad, el cual apareció originalmente en 1993 con el título: Creatings Minds. An Anatomy of Creativity ${ }^{3}$.

De acuerdo con Gardner (2011), en el nivel del campo se siguen los siguientes pasos:

1. Considerar la naturaleza de los sistemas simbólicos con los que trabajaron los creadores.

2. Describir los tipos de prácticas creativas de los individuos en forma de cinco clases diferentes de actividad. Estas actividades también son mencionadas en los interludios. [1. Solución de un problema concreto; 2. Propuesta de un esquema conceptual general; 3. Creación de un producto; 4. Un tipo estilizado de actuación; 5. Una actuación de alto riesgo. Estos cinco tipos de actividades deben ser abordados creativamente, lo que implica “conceptualizar un esquema más complicado, que incluya tres componentes: 1. El sistema (o sistemas) simbólico concreto empleado; 2. La naturaleza de la actividad creativa; 3. Momentos particulares en el curso de un avance o actuación creativos” (pp. 480-481)].

\footnotetext{
${ }^{3}$ Esta obra se tradujo al español por José Pedro Tosaus Abadía y fue publicada en España en 1995 y 2010. En marzo de 2011, fue publicada por primera vez en México, en colaboración con la editorial Paidós de España.
} 
3. Finalmente, es preciso considerar el estatuto de los paradigmas o planteamientos principales, tal y como existen en los campos donde los creadores están trabajando. Se incluye una consideración de la susceptibilidad del paradigma de cara a una innovación continuada a lo largo de la vida del creador.

García y Estebaranz (2005) señalan varios aspectos o características que implica el medio ambiente como "escenario de desarrollo creativo" (pp. 22-23):

- Atmósfera relajada y lúdica.

- Existencia de límites para que surja.

- Equilibrio en las características del trabajo a realizar, el cual debe estar al alcance de los trabajadores.

- La presión en la competición interfiere en la creatividad.

- Los educadores que promueven la creatividad tratan a los alumnos como individuos, fomentan la independencia y sirven de modelos para roles creativos.

- Los entornos que facilitan la creatividad se caracterizan por una buena dirección. Ésta establece metas, evita distracciones y no es demasiado estricta. Además, dispone de recursos suficientes: estimulación de nuevas ideas, colaboración entre las divisiones del trabajo, si las hubiere, reconocimiento del trabajo creativo, disponer de tiempo para pensar, existencia de problemas desafiantes o sentido de urgencia de que el trabajo sea realizado.

- Las sociedades que estimulan la creatividad apoyan el trabajo creativo, por lo que están abiertas a los estímulos culturales. 


\section{BENEFICIOS DEL CUENTO MUSICAL EN EL DESARROLLO DE LA CREATIVIDAD}

De acuerdo con Zelenkova (2010), comprender una obra musical o, como le llama, la imagen de ejecución (p. 238), es una de las tareas más difíciles en la pedagogía musical, pues constituye un concepto abstracto. Sin entrar en la descripción de los procesos que participan en la actividad de la percepción musical infantil, uno de los métodos para acercar al niño al fenómeno musical que describe Zelenkova es la utilización de la creatividad verbal, esto es, la introducción del cuento mágico como una cuestión en donde el niño participe como autor del mismo. Este método de improvisación dirigida, comenta la autora, ha sido utilizado por León Tolstoi (Vigotsky, 1967), Gianni Rodari (1990) y O. M. Dyachenko (1986). El niño mostraba su experiencia más rica en la composición de los cuentos, por encima de la ejecución musical. No obstante, al realizar esta actividad conjunta, conducida por el efecto del contexto, el niño podía ver "la obra musical de tal forma como no se podría hacer con el medio de la percepción usual. El mundo mágico de los cuentos participa aquí como uno de los medios de la representación de la realidad de la vida, como el idioma especial, el cual, al penetrar en la conciencia del alumno, garantiza la creación en él de la imagen de ejecución de la obra musical" (Zelenkova, 2010, pp. 240-241). La música se vuelve la representación simbólica del cuento, el cual sirve de apoyo para la comprensión del mensaje musical, de ahí su interpretación. Zelenkova realizó un experimento con niños de siete a catorce años de edad, quienes estudiaban piano en una escuela de arte. El grupo experimental en el que se utilizó la actividad de creación y narración del cuento, conjuntamente con la interpretación de piano, mostró el incremento en la calidad de ejecución ${ }^{4}$ en su componente creativo, en promedio de 1.77 en una escala usual de 10 niveles, mientras el grupo control no mostró cambios significativos.

Arguedas, como otros investigadores, describe los enormes beneficios que proporciona un cuento musical en la educación del niño, tanto en el ámbito psicomotor como en el socioafectivo y cognitivo, a través de los estímulos literarios y musicales. Dicha experiencia

\footnotetext{
${ }^{4}$ La evaluación de la interpretación musical se dividió en dos indicadores: técnico, que abarca la ejecución correcta de las notas, la precisión y limpieza del sonido, etc. y creativo que incluye la expresión emocional, personalidad del intérprete, aspectos de agógica, dinámica, utilización de matices, manejo del tiempo y pulso.
} 
"motiva la expresión plástica, la expresión dramática y la danza, lo que permite enriquecer la creatividad de las niñas y de los niños" (Arguedas, 2006).

Camino (2012) opina que "no hay duda de que la unión entre la palabra, la música y la imagen produce un resultado mágico con grandes posibilidades educativas" (p.1). En ese mismo tenor, Romero afirma que los "dos ingredientes unidos, palabra y música, son los elementos esenciales para divertir, motivar y desarrollar la imaginación, la creatividad [...]” (Romero, 2007, p. 1) concluyendo así:

El cuento musical conlleva un desarrollo psicomotor, socio-afectivo y cognitivo, ya que mediante éste, se establece una relación con los estímulos musicales, literarios, plásticos, creativos, imaginativos, que incluyen frecuentemente, el movimiento y la danza, lo que servirá como motivación a los niños, poniendo en práctica una enseñanza activa y participativa dentro de lo que consideramos la educación integral. (Romero, 2007, p. 1)

Existen otros trabajos que incluyen propuestas metodológicas para implementar el cuento musical en las actividades del aula, empero, la mayoría de estos textos se dedican nada más a describir diferentes opiniones sobre la importancia de acercar al niño al mundo literario y musical. Al mismo tiempo, describen distintos beneficios que proporciona el cuento musical en el desarrollo de diferentes potenciales del niño, incluyendo la creatividad, su contribución en la formación del infantil de una forma integral, pero prácticamente no se habla de estudios con una base sólida científica que se hayan realizado en este campo.

\section{INVESTIGACIÓN DE CAMPO}

\section{Objetivo}

Al ser un terreno nuevo que aún no ha sido explorado, el objetivo de esta investigación es conocer el efecto del cuento musical que pudiera tener en el desarrollo de la creatividad en los niños de las escuelas primaras a través del empleo de un test de creatividad infantil. 


\section{Hipótesis}

Hipótesis: el cuento musical influye positivamente sobre el desarrollo de la creatividad en niños de escuelas primarias.

ANOVA: Análisis de varianza que proporciona el estadístico F a partir del cual se contrasta la hipótesis nula con una alternativa.

Hipótesis nula: el cuento musical no influye sobre el desarrollo de la creatividad en niños de escuelas primarias. $R 2=0$. Es decir que la variable respuesta (creatividad) no está influida por las variables independientes. Es lo mismo que decir que la variabilidad observada en las respuestas es causada por el azar sin influencia de las variables independientes. En otras palabras, nuestra hipótesis nula significa que el cuento musical no hace ningún cambio en el nivel de la creatividad.

Si el $p$-value asociado al estadístico $\mathrm{F}$ es menor que el nivel de significación (normalmente 0.05), rechazamos la hipótesis nula planteada.

Si la hipótesis nula es rechazada, suponemos que el cuento musical beneficia el desarrollo de la creatividad.

\section{Metodología}

En el presente estudio utilizamos un diseño cuasi-experimental $\mathrm{A}-\mathrm{B}-\mathrm{A}$, con una evaluación inicial (A), la aplicación de un programa integrado por cuentos (B), con 3 variantes (sin música, con música en vivo y con música grabada).

\section{Sujetos}

En el estudio participaron 582 niños de $1^{\circ}$ a $6^{\circ}$ grado de una escuela primaria pública de la ciudad de Puebla. La mayoría de los niños pertenecen a un nivel social bajo o medio-bajo. De los 582 niños, se descartaron 122 niños: 36 participaron en el test piloto y 85 no completaron los dos test (pre-test y post-test), faltando a alguno de éstos. Por añadidura, una alumna no contó con la autorización de sus padres. Por lo tanto, los resultados finales del estudio corresponden a un total de 460 alumnos, con un rango de edad de 6 a 13 años. Los niños de cada grado escolar fueron divididos en 3 grupos, un grupo control y dos grupos experimentales, de acuerdo a las siguientes condiciones: 
Grupo Control: $0(\mathrm{GC})$ - Cuento sin música $(\mathrm{n}=147)$

Grupo Experimental I: 1 (GE-I) - Cuento musical en vivo $(\mathrm{n}=163)$

Grupo Experimental II: 2 (GE-II) - Cuento musical grabado $(\mathrm{n}=150)$

De acuerdo a lo anterior obtuvimos 18 grupos, 6 grupos control, 6 grupos experimentales tipo I y 6 grupos experimentales tipo II.

En la siguiente tabla se observa el número de participantes por grado y tipo de cuento:

Tabla 1. Distribución de sujetos por grado y tipo de cuento.

\begin{tabular}{cccc}
\hline Grado & GC - Sin música & GE1 - Musical en vivo & GE2 - Musical grabado \\
\hline 1 & 25 & 28 & 24 \\
2 & 24 & 25 & 24 \\
3 & 25 & 31 & 29 \\
4 & 31 & 28 & 26 \\
5 & 25 & 28 & 27 \\
6 & 17 & 23 & 20 \\
Total & 147 & 163 & 150 \\
\hline
\end{tabular}

En la siguiente tabla se muestra la distribución de los sujetos por grado y género:

Tabla 2. Distribución de sujetos por grado y género.

\begin{tabular}{cccc}
\hline Grado & Género & & Total \\
& M & F & \\
\hline 1 & 33 & 44 & 77 \\
2 & 39 & 34 & 73 \\
3 & 41 & 44 & 85 \\
4 & 39 & 46 & 85 \\
5 & 37 & 43 & 80 \\
6 & 27 & 33 & 60 \\
Total & 216 & 244 & 460 \\
\hline
\end{tabular}


La asignación del tipo de cuento para cada grupo se hizo al azar. La distribución de grupos y las sesiones (algunas completas, otras parcialmente) fueron videograbadas de esta manera:

1 "B" Cuento sin música (Grupo control)

1 "C" Cuento musical en vivo (Grupo experimental I)

1 "A" Cuento musical grabado (Grupo experimental II)

2 "A" Cuento sin música (Grupo control)

2 "C" Cuento musical en vivo (Grupo experimental I)

2 "B" Cuento musical grabado (Grupo experimental II)

3 "C" Cuento sin música (Grupo control)

3 "B" Cuento musical en vivo (Grupo experimental I)

3 "A" Cuento musical grabado (Grupo experimental II)
4 "B" Cuento sin música (Grupo control)

4 "C" Cuento musical en vivo (Grupo experimental I)

4 "A" Cuento musical grabado (Grupo experimental II)

5 "A" Cuento musical grabado (Grupo experimental II)

5 "B" Cuento musical en vivo (Grupo experimental I)

5 "C" Cuento sin música (Grupo control)

6 "A" Cuento musical grabado (Grupo experimental II)

6 "B" Cuento musical en vivo (Grupo experimental I)

6 "C" Cuento sin música (Grupo control)

\section{Instrumentos}

Antes del curso y al finalizarlo, los niños realizaron una Prueba de creatividad con el fin de evaluar si su participación en las sesiones de cuento había tenido algún impacto en su creatividad. Se utilizó el Test de Creatividad Infantil, de M. Romo Santos, V. Alfonso Benlliure y M. J. Sánchez-Ruiz (2008), investigadores de la Facultad de Psicología de la Universidad Autónoma de Madrid.

Este test fue elegido por varias razones. Los autores del Test de Creatividad Infantil (TCI) nos dejan una descripción detallada sobre su valor, importancia y fundamento teórico. 
Parten de las investigaciones de Getzels y Csikszentmihalyi (1976) e investigaciones de Runco (1994). Por añadidura, este test no solamente es fácil de aplicar, sino que sus resultados son sencillos de interpretar, algo que no se ha podido constatar con otros test de creatividad. Antes de comenzar el estudio, se entregó la información con la descripción del estudio a los padres de familia, solicitándoles su firma de aceptación (Carta de Consentimiento) y el llenado del cuestionario, con el fin de recabar información demográfica de los niños participantes en el estudio.

\section{Descripción de tipos de cuento}

A continuación, se describen los tres tipos de cuentos que fueron impartidos y las actividades realizadas:

- Cuento sin música: son distintos cuentos que fueron leídos y/o contados para niños en cada clase.

- Cuento musical en vivo: son distintos cuentos que fueron leídos o contados con música clásica en vivo que interpreté con la guitarra, interrumpiendo la lectura en determinados momentos del cuento para continuar la idea literaria con la música. Aunque estos cuentos fueron previamente preparados, ya que se tuvo que seleccionar las obras musicales dependiendo del contenido del cuento, había momentos de improvisación en la parte musical.

- Cuento musical grabado: son distintos cuentos musicales grabados en CDs. En cada sesión los niños escucharon distintos cuentos musicales a través de discos.

\section{Test piloto}

Antes de iniciar el estudio se realizó el test de creatividad piloto con el fin de determinar el índice de confiabilidad de la evaluación de las pruebas por parte de tres jurados, alumnos de Licenciatura en Música de la Benemérita Universidad Autónoma de Puebla (BUAP). Los tres evaluadores recibieron el entrenamiento previo y se les explicó el mecanismo de evaluación. De igual forma, se les proporcionó el material y las instrucciones del Manual del Test de Creatividad Infantil. Para la realización del test-piloto se eligieron cuatro niños al 
azar de cada uno de los grupos de $2^{\circ}, 4^{\circ}$ y $6^{\circ}$. Cada uno de los jurados evaluó los 36 test por separado y mostraron un alto grado de concordancia.

La finalidad del test piloto es comprobar que la valoración final de éste sea independiente del evaluador que la realiza, es decir, que el test es fiable y objetivo pues los factores asociados con el evaluador no son relevantes para la valoración final. Lo anterior permite justificar que sólo un jurado evaluará el pre-test y el post-test de cada niño, de cada año escolar, para la tres condiciones del experimento: 0 - cuento sin música, 1 - cuento musical en vivo, 2 - cuento musical grabado.

\section{Resultados del estudio}

En la siguiente tabla se observa los resultados de todos los niños de todos los grados que fueron sometidos a la condición 0: cuento sin música, grupo testigo.

Tabla 3. Puntaje de creatividad de todos los grupos testigo, pre-test y post-test.

\begin{tabular}{ccccc}
\hline $\begin{array}{c}\text { Puntaje } \\
\text { Creatividad }\end{array}$ & Media & $\begin{array}{c}\text { Desviación } \\
\text { Estándar }\end{array}$ & $\begin{array}{c}\text { Error Estándar } \\
\text { de la Media }\end{array}$ & Alumnos \\
\hline Pre-test & 4.81 & 1.86 & 0.15 & 147 \\
Post-test & 4.92 & 1.86 & 0.15 & 147 \\
\hline
\end{tabular}

Tabla 4. Cuento sin música (testigo), todos los grados.

\begin{tabular}{lcccccc}
\hline Hipótesis alternativa & $\mathrm{t}$ & $\mathrm{df}$ & p-value & $\begin{array}{c}\text { intervalo de } \\
\text { confianza de 95\% }\end{array}$ & $\begin{array}{c}\text { media de } \\
\text { diferencias }\end{array}$ \\
\hline $\begin{array}{c}\text { Diferencia de medias } \\
\text { no es igual a 0 }\end{array}$ & 0.63 & 146 & 0.53 & -0.24 & 0.46 & 0.11 \\
\hline
\end{tabular}

El p-value no permite rechazar la hipótesis nula de que la diferencia de medias es igual a 0 .

El cuento sin música no produjo cambio estadísticamente significativo del puntaje promedio de creatividad en este grupo. 
En la siguiente tabla observamos los resultados de los niños de todos los grados que fueron sometidos a la condición 1: cuento musical en vivo, grupo experimental 1.

Tabla 5. Puntaje de creatividad, todos los grupos experimental 1, pre-test y post-test.

\begin{tabular}{ccccc}
\hline $\begin{array}{c}\text { Puntaje } \\
\text { Creatividad }\end{array}$ & Media & $\begin{array}{c}\text { Desviación } \\
\text { Estándar }\end{array}$ & $\begin{array}{c}\text { Error Estándar } \\
\text { de la Media }\end{array}$ & Alumnos \\
\hline Pre-test & 4.00 & 2.09 & 0.16 & 163 \\
Post-test & 4.92 & 2.04 & 0.16 & 163 \\
\hline
\end{tabular}

Tabla 6. Cuento musical en vivo (experimental 1), todos los grados.

\begin{tabular}{ccccccc}
\hline Hipótesis alternativa & $\mathrm{t}$ & $\mathrm{df}$ & p-value & $\begin{array}{c}\text { intervalo de } \\
\text { confianza de 95\% }\end{array}$ & $\begin{array}{c}\text { media de } \\
\text { diferencias }\end{array}$ \\
\hline $\begin{array}{c}\text { Diferencia de medias } \\
\text { no es igual a 0 }\end{array}$ & 4.82 & 162 & $32 \mathrm{e}-06$ & 0.55 & 1.31 & 0.93 \\
$\begin{array}{c}\text { Diferencia de medias } \\
\text { no es igual a 0 }\end{array}$ & 4.82 & 162 & $1.6 \mathrm{e}-06$ & 0.61 & Inf & 0.93 \\
& & & & & & \\
\hline
\end{tabular}

El p-value permite rechazar la hipótesis nula de que la diferencia de medias es igual a 0. El cuento musical en vivo produjo un cambio significativo de puntaje promedio de creatividad en este grupo. Además, en el segundo t-test también podemos rechazar la hipótesis nula, es decir, que el cuento musical en vivo produjo un aumento significativo en el puntaje promedio de creatividad en este grupo.

En la siguiente tabla observamos los resultados de los niños de todos los grados que fueron sometidos a la condición 2: cuento musical grabado, grupo experimental 2. 
Tabla 7. Puntaje de creatividad, todos los grupos experimental 2, pre-test y post-test.

\begin{tabular}{ccccc}
\hline $\begin{array}{c}\text { Puntaje } \\
\text { Creatividad }\end{array}$ & Media & $\begin{array}{c}\text { Desviación } \\
\text { Estándar }\end{array}$ & $\begin{array}{c}\text { Error Estándar } \\
\text { de la Media }\end{array}$ & Alumnos \\
\hline Pre-test & 5.18 & 2.16 & 0.18 & 150 \\
Post-test & 5.91 & 1.86 & 0.15 & 150 \\
\hline
\end{tabular}

Tabla 8. Cuento musical grabado (experimental 2), todos los grados.

\begin{tabular}{ccccccc}
\hline Hipótesis alternativa & $\mathrm{t}$ & $\mathrm{df}$ & p-value & $\begin{array}{c}\text { intervalo de } \\
\text { confianza de } 95 \%\end{array}$ & $\begin{array}{c}\text { media de } \\
\text { diferencias }\end{array}$ \\
\hline $\begin{array}{c}\text { Diferencia de medias } \\
\text { no es igual a 0 }\end{array}$ & 3.84 & 149 & $1.8 \mathrm{e}-04$ & 0.36 & 1.12 & 0.74 \\
$\begin{array}{c}\text { Diferencia de medias } \\
\text { es mayor a 0 }\end{array}$ & 3.84 & 149 & $9.1 \mathrm{e}-05$ & 0.42 & Inf & 0.74 \\
\hline
\end{tabular}

El p-value permite rechazar la hipótesis nula de que la diferencia de medias es igual a 0 . El cuento musical grabado produjo un cambio significativo de puntaje promedio de creatividad en este grupo. Además, en el segundo t-test también podemos rechazar la hipótesis nula, es decir, que el cuento musical grabado produjo un aumento estadísticamente significativo en el puntaje promedio de creatividad en este grupo.

Por otro lado, apreciamos que tanto el cuento musical en vivo como el cuento musical grabado, aunque produjeron un aumento significativo en el puntaje medio de creatividad en este grupo, la significación estadística del cuento musical en vivo representó un orden de magnitud mayor al del cuento grabado (1.6 x e-06 contra 9.1 x e-05). 


\section{Resumen de resultados}

Tabla 9. Incremento estadísticamente significativo de creatividad en el post-test.

\begin{tabular}{cccccccc}
\hline Grados & $1^{\circ}$ & $2^{\circ}$ & $3^{\circ}$ & $4^{\circ}$ & $5^{\circ}$ & $6^{\circ}$ & Todos \\
\hline Sin Música & $\times$ & $\times$ & $\times$ & $\times$ & $\times$ & $\times$ & $\times$ \\
Musical & $\times$ & $\times$ & $\sqrt{ } \sqrt{ }$ & $\times$ & $\sqrt{ } \sqrt{ }$ & $\sqrt{ }$ & $\sqrt{ }$ \\
Grabado & $\sqrt{ } \sqrt{ }$ & $\sqrt{ } \sqrt{ }$ & $X^{*}$ & $\sqrt{ } \sqrt{ }$ & $\sqrt{ } \sqrt{ }$ & $\times$ & $\sqrt{ }$ \\
\hline
\end{tabular}

* Disminuyó el puntaje de creatividad en el post-test.

El cuento sin música no suscitó una variación estadística considerable en el promedio de puntaje de creatividad en ningún grado.

El cuento musical en vivo produjo un cambio estadísticamente significativo del promedio de puntaje de creatividad (el cual se incrementó de forma estadísticamente significativa en el post-test) en los grados $3^{\circ}, 5^{\circ}, 6^{\circ}$ en específico, y al juntar a todos los niños de todos los grados $\left(1^{\circ} \mathrm{al} 6^{\circ}\right)$ que tomaron el test después del cuento musical.

El cuento musical grabado motivó una transformación estadísticamente considerable del promedio de puntaje de creatividad (mismo que se incrementó de forma estadísticamente significativa en el post-test) en los grados $1^{\circ}, 2^{\circ}, 4^{\circ}$ y $5^{\circ}$ en específico, y al juntar a todos los niños de todos los grados $\left(1^{\circ}\right.$ al $\left.6^{\circ}\right)$ que tomaron el test después del cuento musical (el caso específico de $3^{\circ}$ es curioso porque el puntaje de creatividad promedio disminuyó después del cuento grabado).

\section{CONCLUSIONES}

El cuento sin música no produce cambios significativos en el nivel de la creatividad. El cuento musical en vivo y cuento musical grabado aumentan la creatividad.

La presente investigación fue el primer paso para descubrir los beneficios que proporciona un cuento musical en el desarrollo de la creatividad en la escuela primaria. Escuchar y recibir el placer del cuento musical, donde se conjuga tanto el arte literario como el arte musical, es una experiencia valiosa que enriquece la vida de un pequeño, la música y la literatura ayudan a liberar la imaginación del niño y estimulan su potencial creativo. 
Siguiendo los propósitos de la educación artística del "Plan de Estudios de Educación Básica 2011”, que parte de las necesidades y características de los niños, enfocados a fomentar el gusto, la sensibilidad y apreciación hacia las expresiones artísticas y desarrollo de la creatividad mediante la utilización de distintos recursos de las expresiones artísticas, consideramos idóneo, implementación del cuento musical como parte de las actividades de la educación artística en la primaria que sin duda enriquecerá la experiencia del niño y estimulará su imaginación, y de ahí, la creatividad.

De esta manera, se vuelve importante, reconsiderar el tiempo destinado para las disciplinas de la educación artística: artes plásticas, teatro, danza y música que imparten durante una hora a la semana a lo largo de los seis años de la educación primaria.

Si, bien, no se ha podido mostrar que las actividades del cuento sin música eleven la creatividad significativamente, se constata un gran interés por parte de los niños hacia estas actividades que sin duda enriquecen su experiencia y sirven de un valioso estímulo y motivación para hacer de lectura un hábito constante. Asimismo, escuchar un cuento, ayuda a la concentración y atención por parte del niño. Se pudo observar un comportamiento verdaderamente ejemplar por parte de los niños, que escuchaban los cuentos con mucha atención y agrado. Con un replanteamiento de las actividades alrededor de cuentos (que pueden ser explorados conjuntamente con la creación y dramatización de los mismos) y tomando en cuenta el tiempo asignado para la impartición del curso (el estudio tuvo una duración de alrededor de un mes y medio), es posible encontrar más respuestas entorno a la creatividad y el cuento, sus efectos y beneficios. Lo importante, es seguir haciendo este tipo de investigaciones para dar respuestas objetivas, válidas y confiables. Estas respuestas a la vez podrán corresponder a todas las búsquedas, ideas y teorías creadas entorno al beneficio del cuento musical en el desarrollo de la creatividad. La falta de este tipo de estudios que muestren con argumentos válidos el incremento de la creatividad a partir del cuento y la música, nos obligan a seguir estudiando este tema, realizar mayor número de estudios con las actividades y planeación correspondientes, tomando también en cuenta las tendencias de una nueva época y sus necesidades.

Todos los seres humanos, de alguna u otra forma, tienen el potencial creativo y éste, a su vez, puede ser desarrollado. Propiciar, favorecer y ofrecer oportunidades de desarrollo de 
dicho potencial, en un ambiente de respeto y aceptación, debe ser primordial para cualquier profesor, sobre todo en la educación primaria, porque desde estas edades se siembra la semilla y se generan las oportunidades para todas las experiencias que formarán una base sólida en el crecimiento de los individuos y, sobre todo, en el desarrollo de su creatividad.

El tema de la creatividad es un tema muy diverso y complejo y, a la vez, fascinante. Debemos seguir trabajando unidos en el desarrollo de nuevas propuestas e iniciativas para una enseñanza creativa que, sin duda, va a beneficiar a nuestros niños en todos los sentidos como seres humanos.

La escuela debe enfocar sus actividades en el desarrollo de la creatividad, contribuir a la formación de personas creativas y críticas, capaces de expresar sus ideas de forma oral, escrita o de manera artística; el espacio idóneo es la educación artística que debe ocupar un lugar privilegiado dentro del currículo escolar, con maestros especializados en el campo artístico que a la vez podrán responder a las exigencias del tiempo moderno, de las tendencias contemporáneas y a los propósitos y metas que establece la Secretaría de Educación Pública a través de distintos documentos que ha elaborado en los últimos quince años. De igual forma, la academia está obligada a crear espacios y condiciones para el desarrollo de la creatividad. Paralelamente, debe actualizar y revisar los programas de estudio de la educación artística, implementando nuevas propuestas e ideas y explorando nuevos enfoques hacia una educación creativa. 


\section{Bibliografía}

Arguedas Quesada, C. (2006). Cuentos musicales para los más pequeños. Actualidades Investigativas en Educación, 6 (1). Recuperado de http://revista.inie.ucr.ac.cr/uploads/ tx_magazine/cuentossss.pdf [2015, 23 de febrero].

Bertomeu Orteu, C. (2006). El cuento. Un medio para adentrarnos en los secretos de la obra artística. En B. López Romero (Ed.), El cuento como instrumento para el desarrollo de la

Camino, M. J. (2012, mayo 11). La magia de contar un cuento con música: Pedro y el Lobo [Mensaje en el blog Educ@ contic]. Recuperado de http://www.educacontic.es/blog/la-magia-de-contar-un-cuento-con-musica-pedro-yel-lobo [2015, 23 de febrero].

Campbell, D. (2007). El efecto Mozart para niños. Despertar con música el desarrollo y la creatividad de los más pequeños (Trad. Brito, A.). Barcelona: Urano (1997).

Campbell, D. (2011). El efecto Mozart. Barcelona: Urano (4a ed.).

Copland, A. (2003). Música e imaginación (Trad. Ortiz Oderigo, N. R.). Buenos Aries. Emecé Editores (1952).

Csikszentmihalyi (1997). Fluir (Flow). Una psicología de la felicidad. Barcelona: Kairós.

Csikszentmihalyi, M. (1998). Creatividad. El fluir y la psicología del descubrimiento y la invención (Trad. Tosaus Abadía, J. P.). Barcelona: Paidós (1996).

Finke, R., Ward, T. \& Smith, S. (1992). Creative cognition. Cambridge Mass.: The MIT Press.

Firestein, R. (1991). Creatividad en la Empresa. Conferencia Fundación Creatividad e Innovación, Santiago. 
Frega, A. L. (2009). Creatividad y Educación Musical. Revista Creatividad y Sociedad, (10), 9-31.

García Calero P. \& Estebaranz García A. (2005). Innovación y creatividad en la enseñanza musical. Barcelona: Octaedro.

Gardner, H. (2011) Mentes Creativas. Una anatomía de la creatividad (Trad. Tosaus Abadía, J. P.). Barcelona: Paidós (1993).

Getzels, J. M. \& Csikszentmihalyi, M. (1976). The Creative Vision. A Longitudinal Study of Problem Finding in Art. New York: J. Willey and Sons.

Gruber, H. E. (1984). Darwin sobre el hombre: un estudio psicológico de la creatividad científica (Trad. Amo, T. d.). Madrid: Alianza, (1974).

Hargreaves, D. J. (1998). Música y desarrollo psicológico (Trads. Frega, A. L., Graetzer, D. \& Musumeci, O.). Barcelona: Graó (1986).

López de la Llave, A. \& Pérez, M. (2006). Psicología para intérpretes artísticos. Madrid: Thomson / Paraninfo.

López Pérez, R. (1999). Prontuario de la Creatividad. Recuperado de http://mcomudd.files. wordpress.com/2008/01/r-lopez-prontuario-creatividad.pdf [2015, 24 de febrero].

López Romero, B. (2006a). El cuento, vehículo de transmisión de conceptos y valores sociales. En B. López Romero (Ed.), El cuento como instrumento para el desarrollo de la creatividad artística. España: Ministerio de Educación y Cultura.

MacKinnon, D. W. (1975). IPAR's contribution to the conceptualization and study of creativity. En Getzels, J. \& Taylor, I. A., Perspectives in creativity. Chicago: Aldine.

Maslow A. (1982). La personalidad creadora (Trad. Rourich, R. M.). Barcelona: Kairós (1972).

Newell, A., Shaw, J. C. \& Simon, H. A. (1958). Elements of a theory of human problem solving. Psychological Review, 65 (3), 151-166. 
Orff-Schulwerk (1969). Música para niños, introducción. Editorial: Unión Musical Española.

Rodríguez Estrada, M. (2009). Manual de creatividad. México: Editorial Trillas.

Romero Chaparro, M. E. (2007). Los cuentos musicales en la educación primaria. Filomúsica. Revista mensual de publicación en Internet, (82) Recuperado de http://www.filomusica. com/filo82/cuentos.html [2015, 24 de febrero].

Romo, M. (1997). Psicología de la creatividad. Barcelona: Paidós.

Romo, M., Benlliure, A. \& Sánchez-Ruiz, M. J. (2008). Test de Creatividad Infantil. Madrid: TEA Ediciones.

Runco, M. A. (1994). Problem Finding, Problem Solving and Creativity. New York: Ablex Publishing Corporation.

Tatarkiewicz, W. (1987), Historia de seis ideas: arte, belleza, forma, creatividad, mimesis, experiencia estética (Trad. Rodríguez Martín, F.). Madrid: Tecnos.

Torrance, E. P. (1970). Desarrollo de la creatividad del alumno (Trad. Schwarz, R. E.). Buenos Aires: Centro Regional de Ayuda Técnica.

Vigotsky, L. S. (2008). Voobrazheniye y tvorchestvo $v$ detskom vozraste (La imaginación y la creatividad en la edad infantil). En Mishleniye y rech (Pensamiento y el lenguaje). Moscú: ACT MOSKVA.

Vigotsky, L. S. (1984) Problemas de psicología infantil. Obras escogidas, Tomo IV. Moscú: Pedagogía.

Waisburg, G. \& Erdmenger, E. (2008). El poder de la Música en el aprendizaje: cómo lograr un aprendizaje acelerado y creativo. México: Trillas.

Wilson, B. G. (1979). Evaluación del Aprendizaje en la Educación Artística. En B. S. Bloom, J. T. Hastings \& G. F. Madaus (Coords). Evaluación del Aprendizaje 3. 
Educación Artística, Ciencia, Matemática de la Escuela Secundaria (Trad. Mizraji, M. N. \& Walton, R. J.). Buenos Aires: Editorial Troquel.

Wollschlager, G. (1976). Creatividad, sociedad y educación. Barcelona: Promoción Cultural.

Zelenkova, T. V. (2010). El desarrollo de la imaginación creativa en los niños durante la enseñanza de la música. En Y. Solovieva \& L. Quintanar (Eds.), Antología del desarrollo psicológico del niño en edad preescolar (pp. 235-255). México: Editorial Trillas. 